\title{
Stability Analysis of a Fractional-Order Differential Equation System of a GBM-IS Interaction Depending on the Density
}

\author{
Fatma Bozkurt* \\ Mathematics Department, Erciyes University, 38039 Kayseri, Turkiye \\ Received: 12 May. 2013, Revised: 16 Sep. 2013, Accepted: 17 Sep. 2013 \\ Published online: 1 May. 2014
}

\begin{abstract}
In this paper, fractional-order is introduced into the interaction model between GBM and IS. GBM (Glioblastoma Multiforme) is a brain tumor, that has a monoclonal origin and produces after reaching a specific density another tumor with different growth rate and treatment susceptibilities. The IS cells are also divided into two populations, namely, the macrophages and the activated macrophages. Hence, this model show two conversions, the conversion from sensitive tumor cell to resistant tumor cell and the conversion from macrophages to active macrophages, and an interaction between the tumor cell and the macrophages. In this work, it is shown that the model possesses non-negative solutions. Furthermore, the stability, existence and uniqueness were studied. To investigate the conditions for an extinction of the tumor cells, Allee threshold is considered. Numerical simulations will give a detailed description of the behavior of the constructed models at the end of the paper.
\end{abstract}

Keywords: Fractional order differential equation, stability, existence, uniqueness, Allee threshold

\section{Introduction}

Cancer is one of the greatest killer in the world and the control of tumor growth requires special attention [1]. The typical approach for treating GBM involves surgical resection of as much of the tumor as possible, followed by radiation treatment and chemotherapy [2]. Within a single tumor of monoclonal origin, this kind of tumor can develop multiple sub-populations, each of which may be characterized by different growth-rates and treatment susceptibilities $[3-5]$. Works about modeling of multi sub- populations can be shown in $[6-10]$. Modeling the interaction between the tumor cell and the immune system are interesting researches, where a few of them will be referee, see $[1,11-13]$. However, a large amount of work done on modeling tumor growth has been restricted to integer order ordinary or delay differential equations [14-17]. Recently, fractional calculus has been applied in many fields $[18-22,32,33]$. In biology, it has been deduced that the membranes of cells of biological organism have fractional order electrical conductance [23]. Fractional derivatives embody essential features of cell rheological behavior and have enjoyed success in the field of rheology [24]. It is shown that modeling the behavior of brainstem vestibule-oculumotor neurons by fractional order differential equation has more advantages than by integer order differential equation [21]. In this paper GBM has two kind of cells, the sensitive tumor cell and resistant tumor cell. Additionally, for the IS we will consider the activated macrophages and macrophages. Thus, taking into account the converting of the sensitive tumor cell to resistant tumor cell, the converting of the macrophages to the activated macrophages and the interaction between the tumor cells and the activated macrophages, we construct a fractional-order the model such as

$$
\left\{\begin{array}{l}
D^{\alpha}(S(t))=p S(t)+r_{1} S(t)\left(K_{1}-\beta_{1} S(t)\right)-\gamma S(t) R(t) \\
-d_{1} S(t)-\tau_{1} S(t) N(t) \\
D^{\alpha}(R(t))=r_{2} R(t)\left(K_{2}-\beta_{2} R(t)\right)+\gamma S(t) R(t) \\
-d_{2} R(t)-\tau_{2} R(t) N(t) \\
D^{\alpha}(N(t))=\mu N(t) Z(t)-\varepsilon Z(t) N(t)-d_{3} N(t) \\
D^{\alpha}(Z(t))=r_{3} Z(t)\left(K_{3}-\delta_{1} Z(t)\right)-\mu N(t) Z(t) \\
+\varepsilon Z(t) N(t)-d_{4} Z(t),
\end{array}\right.
$$

\footnotetext{
${ }^{*}$ Corresponding author e-mail: fbozkurt@erciyes.edu.tr
} 
where $\mathrm{t} \geq 0$, the parameters $\beta_{1}, \beta_{2}, \delta_{1}, \tau_{1}, \tau_{2}, \gamma, \mu, \varepsilon, p$, $d_{1}, d_{2}, d_{3}, d_{4}, K_{1}, K_{2}, K_{3}, r_{1}, r_{2}$ and $r_{3}$ denote positive numbers. GBM is a brain tumor, that has at least two population. Here, we will have the sensitive tumor cell and resistant tumor cell, which is given in system (1) with $S(t)$ and $R(t)$, respectively. $N(t)$ and $Z(t)$ show the activated macrophages and macrophages, respectively. $p$ is the division rate of the sensitive tumor cell, $K_{1}$ and $K_{2}$ are the carrying capacities of the sensitive (including negrotic part) and resistant cell, respectively. Parameter $\beta_{1}$ and $\beta_{2}$ are necessary to construct logistic differential equations. $r_{1}$ and $r_{2}$ are the growth rate of the sensitive cell and resistant cell, respectively. $\gamma$ is the converting rate of sensitive tumor cell to resistant tumor cell. The parameter $d_{1}$ and $d_{2}$ are their dead rate caused from drugs, respectively. $\mu$ is the conversion rate of macrophages to active macrophages. $d_{3}$ is the natural death of active macrophages and $d_{4}$ natural death of macrophages. $r_{3}$ is the growth rate of macrophages and the parameter $\delta_{1}$ is selected in view of logistic differential equations. $K_{3}$ gives the carrying capacity of macrophages. $\varepsilon$ is the conversion rate of the activated macrophages to macrophages. $\tau_{1}$ and $\tau_{2}$ are the destroying rate caused from the activated macrophages. In section 2, we show that (1) has non-negative equilibrium points and analyze the stability of these steady states. We have investigated the existence and uniqueness of the fractional order model in section 3 . Since we obtain in section 2 , that $\left(0,0, B, \frac{d_{3}}{(\mu-\varepsilon)}\right)$ is an unstable steady state, we modify system (1) in section 4 to get the stability conditions of the threshold. Obtaining a solution that is less than the threshold leads to an extinction that is desired in the population dynamics. Numerical simulations will give a detailed description of the behavior of system (1) at the end of the paper.

Definition 1.1. [25] The fractional integral of order $\alpha>0$ of a function $f: R^{+} \rightarrow R$ is given by

$$
I_{0}^{\alpha} f(x)=\frac{1}{\Gamma(\alpha)} \int_{0}^{x}(x-t)^{\alpha-1} f(t) d t
$$

provided the right side is pointwise defined on $R^{+}$.

Definition 1.2. [25] The Caputo fractional derivative of order $\alpha \in(n-1, n)$ of a continuous function $f: R^{+} \rightarrow R$ is given by

$$
D^{\alpha} f(x)=I_{0}^{n-\alpha} D^{n} f(x), D=\frac{d}{d t}
$$

\section{Stability Analysis}

\subsection{Equilibrium points}

Let us consider the system

$$
\left\{\begin{array}{l}
D^{\alpha}(S(t))=f(S(t), R(t), N(t), Z(t))=p S(t)-d_{1} S(t) \\
+r_{1} S(t)\left(K_{1}-\beta_{1} S(t)\right)-\gamma S(t) R(t)-\tau_{1} S(t) N(t) \\
D^{\alpha}(R(t))=g\left(S(t), R(t), N(t), Z(t)=-\tau_{2} R(t) N(t)\right. \\
+r_{2} R(t)\left(K_{2}-\beta_{2} R(t)\right)+\gamma S(t) R(t)-d_{2} R(t) \\
D^{\alpha}(N(t))=h(S(t), R(t), N(t), Z(t))=\mu N(t) Z(t) \\
-\varepsilon Z(t) N(t)-d_{3} N(t) \\
D^{\alpha}(Z(t))=k(S(t), R(t), N(t), Z(t))=-\mu N(t) Z(t) \\
+r_{3} Z(t)\left(K_{3}-\delta_{1} Z(t)\right)+\varepsilon Z(t) N(t)-d_{4} Z(t),
\end{array}\right.
$$

We want to discuss the stability analysis of (4). Let us perturb the equilibrium point by adding positive terms $\varepsilon_{1}(t), \varepsilon_{2}(t), \varepsilon_{3}(t)$ and $\varepsilon_{2}(t)$, that is

$$
\begin{gathered}
S(t)-\bar{S}=\varepsilon_{1}(t), R(t)-\bar{R}=\varepsilon_{2}(t), \\
N(t)-\bar{N}=\varepsilon_{3}(t) \text { and } Z(t)-\bar{Z}=\varepsilon_{4}(t) .
\end{gathered}
$$

Then we have

$$
\begin{aligned}
& D^{\alpha}\left(\varepsilon_{1}(t)\right) \simeq \quad f(\bar{S}, \bar{R}, \bar{N}, \bar{Z}) \quad+\quad \frac{\partial f(\bar{S}, \bar{R}, \bar{N}, \bar{Z})}{\partial S} \varepsilon_{1}(t) \\
& +\frac{\partial f(\bar{S}, \bar{R}, \bar{N}, \bar{Z})}{\partial R} \varepsilon_{2}(t)+\frac{\partial f(\bar{S}, \bar{R}, \bar{N}, \bar{Z})}{\partial N} \varepsilon_{3}(t)+\frac{\partial f(\bar{S}, \bar{R}, \bar{N}, \bar{Z})}{\partial Z} \varepsilon_{4}(t), \\
& D^{\alpha}\left(\varepsilon_{2}(t)\right) \simeq g(\bar{S}, \bar{R}, \bar{N}, \bar{Z})+\frac{\partial g(\bar{S}, \bar{R}, \bar{N}, \bar{Z})}{\partial S} \varepsilon_{1}(t) \\
& +\frac{\partial g(\bar{S}, \bar{R}, \bar{N}, \bar{Z})}{\partial R} \varepsilon_{2}(t)+\frac{\partial f(\bar{S}, \bar{R}, \bar{N}, \bar{Z})}{\partial N} \varepsilon_{3}(t)+\frac{\partial g(\bar{S}, \bar{R}, \bar{N}, \bar{Z})}{\partial Z} \varepsilon_{4}(t), \\
& \quad D^{\alpha}\left(\varepsilon_{3}(t)\right) \simeq h(\bar{S}, \bar{R}, \bar{N}, \bar{Z})+\frac{\partial h(\bar{S}, \bar{R}, \bar{N}, \bar{Z})}{\partial S} \varepsilon_{1}(t) \\
& +\frac{\partial h(\bar{S}, \bar{R}, \bar{N}, \bar{Z})}{\partial R} \varepsilon_{2}(t)+\frac{\partial h(\bar{S}, \bar{R}, \bar{N}, \bar{Z})}{\partial N} \varepsilon_{3}(t)+\frac{\partial f(\bar{S}, \bar{R}, \bar{N}, \bar{Z})}{\partial Z} \varepsilon_{4}(t) \\
& \text { and } \quad \simeq \quad \frac{\partial k(\bar{S}, \bar{R}, \bar{N}, \bar{Z})}{\partial S} \varepsilon_{1}(t) \\
& D^{\alpha}\left(\varepsilon_{4}(t)\right) \\
& +\frac{\partial k(\bar{S}, \bar{R}, \bar{R}, \bar{Z})}{\partial R} \varepsilon_{2}(t)+\frac{\partial k(\bar{N}, \bar{R}, \bar{N}, \bar{Z})}{\partial N} \varepsilon_{3}(t)+\frac{\partial f(\bar{S}, \bar{R}, \bar{N}, \bar{Z})}{\partial Z} \varepsilon_{4}(t) .
\end{aligned}
$$

We used the fact that $f(\bar{S}, \bar{R}, \bar{N}, \bar{Z})=g(\bar{S}, \bar{R}, \bar{N}, \bar{Z})=$ $h(\bar{S}, \bar{R}, \bar{N}, \bar{Z})=k(\bar{S}, \bar{R}, \bar{N}, \bar{Z})=0$ and obtain therefore a linearized system about the equilibrium point such as

$$
D^{\alpha}(U)=J U,
$$

where $U=\left(\varepsilon_{1}(t), \varepsilon_{2}(t), \varepsilon_{3}(t), \varepsilon_{4}(t)\right)$ and $\mathrm{J}$ is the Jacobian matrix evaluated at the equilibrium. We have $B^{-1} J B=C$, where $\mathrm{C}$ is a diagonal matrix of $\mathrm{J}$ given by

$$
C=\left[\begin{array}{cccc}
\lambda_{1} & 0 & 0 & 0 \\
0 & \lambda_{2} & 0 & 0 \\
0 & 0 & \lambda_{3} & 0 \\
0 & 0 & 0 & \lambda_{4}
\end{array}\right]
$$

where $\lambda_{1}, \lambda_{2}, \lambda_{3}$ and $\lambda_{4}$ are the eigenvalues and $\mathrm{B}$ the eigenvectors of J. Therefore, we get

$$
\left\{\begin{array}{l}
D_{*}^{\alpha}\left(\eta_{1}\right)=\lambda_{1} \eta_{1} \\
D_{*}^{\alpha}\left(\eta_{2}\right)=\lambda_{2} \eta_{2}, \\
D_{*}^{\alpha}\left(\eta_{3}\right)=\lambda_{3} \eta_{3} \\
D_{*}^{\alpha}\left(\eta_{4}\right)=\lambda_{4} \eta_{4},
\end{array}\right.
$$


where $\eta=\left[\begin{array}{l}\eta_{1} \\ \eta_{2} \\ \eta_{3} \\ \eta_{4}\end{array}\right]$ and $\eta=B^{-1} Z$, and whose solutions are given by Mittag-Leffler functions

$$
\eta_{1}(t)=\sum_{n=i}^{\infty} \frac{t^{n \alpha} \lambda_{i}{ }^{n}}{\Gamma(n \alpha+1)} \eta_{1}(0)=E_{\alpha}\left(\lambda_{i} t^{\alpha}\right) \eta_{1}(0)
$$

where $i=0,1,2,4$.

By using the result of Matignon [26], if $\left|\arg \left(\lambda_{i}\right)\right|>\frac{\alpha \pi}{2}(i=1,2,3,4)$, then $\eta_{i}(t)$ are decreasing and therefore are also $\varepsilon_{i}(t)$ decreasing. Thus, let the solution $\left(\varepsilon_{1}(t), \varepsilon_{2}(t), \varepsilon_{3}(t), \varepsilon_{4}(t)\right)$ of $(7)$ exist. If the solution of (7) is increasing, then $(\bar{S}, \bar{R}, \bar{N}, \bar{Z})$ is unstable and if $\left(\varepsilon_{1}(t), \varepsilon_{2}(t), \varepsilon_{3}(t), \varepsilon_{4}(t)\right)$ is decreasing, then the equilibrium point $(\bar{S}, \bar{R}, \bar{N}, \bar{Z})$ is locally asymptotically stable.

Now we want to obtain the equilibrium points of system study the equilibrium and stability of system (4). Considering (4), we want to consider the following equilibrium points, which are biological admissible.

$$
\Lambda_{1}=\left(0,0, B, \frac{d_{3}}{\mu-\varepsilon}\right)
$$

where $\mu>\varepsilon$ and

$\Lambda_{2}=\left(\frac{r_{2} \beta_{2}\left(p+r_{1} K_{1}-d_{1}-\tau_{1} B\right)-\gamma\left(r_{2} K_{2}-d_{2}-\tau_{2} B\right)}{r_{1} r_{2} \beta_{1} \beta_{2}+\gamma^{2}}\right.$,

$$
\left.\frac{\beta_{1} r_{1}\left(r_{2} K_{2}-d_{2}-\tau_{2} B\right)+\gamma\left(p+r_{1} K_{1}-d_{1}-\tau_{1} B\right)}{r_{1} r_{2} \beta_{1} \beta_{2}+\gamma^{2}}, B, \frac{d_{3}}{\mu-\varepsilon}\right)
$$

where

$$
B=\frac{\left(r_{3} K_{3}-d_{4}\right)(\mu-\varepsilon)-r_{3} \delta_{1} d_{3}}{(\mu-\varepsilon)^{2}}
$$

If

$$
r_{3}>\frac{(\mu-\varepsilon) d_{4}}{K_{3}(\mu-\varepsilon)-\delta_{1} d_{3}} \text { and } K_{3}>\frac{\delta_{1} d_{3}}{\mu-\varepsilon},
$$

then we have $B>0$. If we have the conditions

$$
r_{1}>\frac{d_{1}+\tau_{1} B}{K_{1}} a n d r_{1}>\frac{d_{2}+\tau_{2} B}{K_{2}},
$$

where $p>\frac{\gamma K_{2}}{\beta_{2}}$ the equilibrium point $\Lambda_{2}$ is positive.

\subsection{Local Stability}

The Jacobian matrix $J\left(\Lambda_{1}\right)$ for system (4) evaluated at $\Lambda_{1}$ show us that for the conditions (13), (14) and (15) $\Lambda_{1}$ is unstable. To discuss the local stability of the equilibrium point $\Lambda_{2}$, this means that both tumors exist in the brain, we consider the linearized system of (4) at $\Lambda_{2}$. Considering the Jocabian matrix $J\left(\Lambda_{2}\right)$ for system (4), we obtain the characteristic equation of (4) such as

$$
\begin{aligned}
& P(\lambda)=\left(\lambda^{2}-a_{44} \lambda-a_{43} a_{34}\right) \\
& \left(\lambda^{2}-\left(a_{11}+a_{22}\right) \lambda+a_{11} a_{22}-a_{12} a_{21}\right)=0,
\end{aligned}
$$

where

$$
\begin{aligned}
& a_{11}=p+r_{1} K_{1}-d_{1}-2 r_{1} \beta_{1} \bar{S}-\gamma \bar{R}-\tau_{1} \bar{N}, \\
& a_{22}=r_{2} K_{2}-d_{2}-2 r_{2} \beta_{2} \bar{R}+\gamma \bar{S}-\tau_{2} \bar{N}, \\
& a_{44}=\frac{\left(r_{3} K_{3}-d_{4}-(\mu-\varepsilon) B\right)(\mu-\varepsilon)-2 r_{3} \delta_{1} d_{3}}{(\mu-\varepsilon)} \\
& a_{12}=-\gamma \bar{S}, a_{21}=\gamma \bar{R}, a_{34}=(\mu-\varepsilon) B, a_{43}=-d_{3} .
\end{aligned}
$$

Considering (16), we can obtain the following theorem.

Theorem 2.1. Let $\Lambda_{2}$ be the positive equilibrium point of system (4). The following statements are true.

(a) Assume that $K_{3}>\frac{2 \delta_{1} d_{3}}{\mu-\varepsilon}, K_{2}<\frac{\left(d_{2}+\tau_{2} B\right) \beta_{2}}{\gamma}$ and $\frac{\gamma}{\beta_{1}}>\frac{r_{2} K_{2}-d_{2}-\tau_{2} B}{p+r_{1} K_{1}-d_{1}-\tau_{1} B}$ hold. If

$$
r_{2}>\frac{d_{2}+\tau_{2} B}{K_{2}}
$$

and

$$
\frac{d_{4}(\mu-\varepsilon)}{K_{3}(\mu-\varepsilon)-\delta_{1} d_{3}}<r_{3}<\frac{d_{4}(\mu-\varepsilon)+(\mu-\varepsilon)^{2} B}{K_{3}(\mu-\varepsilon)-2 \delta_{1} d_{3}},
$$

then all roots of (16) have are real or complex conjugates with negative real parts, which means that $\Lambda_{2}$ is locally asymptotically stable.

(b) Assume that $K_{3}>\frac{2 \delta_{1} d_{3}}{\mu-\varepsilon}, K_{2}>\frac{\left(d_{2}+\tau_{2} B\right) \beta_{2}}{\gamma}$ and

$$
\begin{gathered}
M \in\left(0, \sqrt{\frac{r_{2} \beta_{2}\left(2 r_{1} r_{2} \beta_{1} \beta_{2} \gamma+4 \gamma^{3}-r_{1}^{2} r_{2} \beta_{1}^{2} \beta_{2}-r_{2} \beta_{2} \gamma^{2}\right)}{r_{1} \beta_{1}\left(2 r_{1} r_{2} \beta_{1} \beta_{2} \gamma+4 \gamma^{3}+r_{1} r_{2}^{2} \beta_{1} \beta_{2}^{2}+r_{1} \beta_{1} \gamma^{2}\right)}}\right) \\
\cup\left(\frac{r_{2} \beta_{2}\left(r_{1} \beta_{1}+\gamma\right)}{r_{1} \beta_{1}\left(-r_{2} \beta_{2}+\gamma\right)}, \infty\right)
\end{gathered}
$$

hold, where

$$
M=\frac{r_{2} K_{2}-d_{2}-\tau_{2} B}{p+r_{1} K_{1}-d_{1}-\tau_{1} B} .
$$

Furthermore, suppose that

$$
\left|\tan ^{-1}\left(-\sqrt{4\left(a_{11} a_{22}-a_{12} a_{21}\right)-\left(a_{11}+a_{22}\right)^{2}}\right) /\left(a_{11}+a_{22}\right)\right|>\frac{\alpha \pi}{2} .
$$

If

$$
\frac{\gamma}{\beta_{2}}>r_{2}>\frac{d_{2}+\tau_{2} B}{K_{2}}
$$

and

$$
\frac{d_{4}(\mu-\varepsilon)+(\mu-\varepsilon)^{2} B}{K_{3}(\mu-\varepsilon)-\delta_{1} d_{3}}<r_{3}<\frac{d_{4}(\mu-\varepsilon)+(\mu-\varepsilon)^{2} B}{K_{3}(\mu-\varepsilon)-2 \delta_{1} d_{3}},
$$

then (16) has has two real or complex conjugates with negative real parts and two complex conjugate with positive real parts, where $\Lambda_{2}$ is locally asymptotically stable.

(c) Assume that $K_{2}<\frac{\left(d_{2}+\tau_{2} B\right) \beta_{2}}{\gamma}, K_{3}>\frac{4 \delta_{1} d_{3}}{\mu-\varepsilon}$, $\frac{\mu-\varepsilon}{r_{3} \delta_{1}}>\sqrt{\frac{d_{3}}{(\mu-\varepsilon) B}}$ and $\frac{\gamma}{\beta_{1}}>\frac{r_{2} K_{2}-d_{2}-\tau_{2} B}{p+r_{1} K_{1}-d_{1}-\tau_{1} B}$ hold.

Furthermore, suppose that we have

$$
\left|\tan ^{-1}\left(-\sqrt{-4\left(a_{43} a_{34}-a_{44}^{2}\right) / a_{44}}\right)\right|>\frac{\alpha \pi}{2} .
$$


If

$$
r_{2}>\frac{d_{2}+\tau_{2} B}{K_{2}}
$$

and

$$
\frac{d_{4}(\mu-\varepsilon)+(\mu-\varepsilon)^{2} B}{K_{3}(\mu-\varepsilon)-2 \delta_{1} d_{3}}<r_{3}<\frac{d_{4}(\mu-\varepsilon)+(\mu-\varepsilon)^{2} B}{K_{3}(\mu-\varepsilon)-4 \delta_{1} d_{3}},
$$

then (16) has has two real or complex conjugates with negative real parts and two complex conjugate with positive real parts, where $\Lambda_{2}$ is locally asymptotically stable.

(d) Assume that $K_{2}>\frac{\left(d_{2}+\tau_{2} B\right) \beta_{2}}{\gamma}, K_{3}>\frac{4 \delta_{1} d_{3}}{\mu-\varepsilon}$, $\frac{\mu-\varepsilon}{r_{3} \delta_{1}}>\sqrt{\frac{d_{3}}{(\mu-\varepsilon) B}}$ and

$$
M \in\left(0, \sqrt{\frac{r_{2} \beta_{2}\left(2 r_{1} r_{2} \beta_{1} \beta_{2} \gamma+4 \gamma^{3}-r_{1}^{2} r_{2} \beta_{1}^{2} \beta_{2}-r_{2} \beta_{2} \gamma^{2}\right)}{r_{1} \beta_{1}\left(2 r_{1} r_{2} \beta_{1} \beta_{2} \gamma+4 \gamma^{3}+r_{1} r_{2}^{2} \beta_{1} \beta_{2}^{2}+r_{1} \beta_{1} \gamma^{2}\right)}}\right)
$$

$$
\cup\left(\frac{r_{2} \beta_{2}\left(r_{1} \beta_{1}+\gamma\right)}{r_{1} \beta_{1}\left(-r_{2} \beta_{2}+\gamma\right)}, \infty\right)
$$

hold, where

$$
M=\frac{r_{2} K_{2}-d_{2}-\tau_{2} B}{p+r_{1} K_{1}-d_{1}-\tau_{1} B} .
$$

Furthermore, suppose that $\alpha$ is in the interval satisfying

$$
\left|\tan ^{-1}\left(-\sqrt{4\left(a_{11} a_{22}-a_{12} a_{21}\right)-\left(a_{11}+a_{22}\right)^{2}}\right) /\left(a_{11}+a_{22}\right)\right|
$$

$>\frac{\alpha \pi}{2}$. and

$$
\left|\tan ^{-1}\left(-\sqrt{-4\left(a_{43} a_{34}-a_{44}^{2}\right) / a_{44}}\right)\right|>\frac{\alpha \pi}{2} .
$$

If

$$
\frac{\gamma}{\beta_{2}}>r_{2}>\frac{d_{2}+\tau_{2} B}{K_{2}}
$$

and

$$
\frac{d_{4}(\mu-\varepsilon)+(\mu-\varepsilon)^{2} B}{K_{3}(\mu-\varepsilon)-2 \delta_{1} d_{3}}<r_{3}<\frac{d_{4}(\mu-\varepsilon)+(\mu-\varepsilon)^{2} B}{K_{3}(\mu-\varepsilon)-4 \delta_{1} d_{3}},
$$

then all of the roots of (16) are complex conjugates with positive real parts, which implies that $\Lambda_{2}$ is locally asymptotically stable.

Proof. The proof follows by using the theory in [27] and will be omitted.

\section{Existence and Uniqueness}

Consider system (4) with the initial conditions $S(0)>0$, $R(0)>0, N(0)>0$ and $Z(0)>0$. The initial value problem can be written in the form

$$
D^{\alpha} U(t)=A U(t)+S(t) B U(t)+R(t) C U(t)+N(t) D U(t)
$$

$$
+Z(t) E U(t), t \in(0, T]
$$

and $U(0)=U_{0}$, where

$$
\begin{gathered}
U(t)=\left[\begin{array}{l}
S(t) \\
R(t) \\
N(t) \\
Z(t)
\end{array}\right], U(0)=\left[\begin{array}{c}
S(0) \\
R(0) \\
N(0) \\
Z(0)
\end{array}\right], \\
A=\left[\begin{array}{cccc}
p-d_{1}+r_{1} K_{1} & 0 & 0 & 0 \\
0 & r_{2} K_{2}-d_{2} & 0 & 0 \\
0 & 0 & -d_{3} & 0 \\
0 & 0 & 0 & 0
\end{array}\right],
\end{gathered}
$$

$$
B=\left[\begin{array}{cccc}
-r_{1} \beta_{1} & 0 & 0 & 0 \\
0 & \gamma & 0 & 0 \\
0 & 0 & 0 & 0 \\
0 & 0 & 0 & 0
\end{array}\right], C=\left[\begin{array}{cccc}
-\gamma & 0 & 0 & 0 \\
0 & r_{2} \beta_{2} & 0 & 0 \\
0 & 0 & 0 & 0 \\
0 & 0 & 0 & 0
\end{array}\right]
$$

$D=\left[\begin{array}{cccc}-\tau_{1} & 0 & 0 & 0 \\ 0 & -\tau_{2} & 0 & 0 \\ 0 & 0 & 0 & 0 \\ 0 & 0 & 0 & -(\mu-\varepsilon)\end{array}\right], E=\left[\begin{array}{cccc}0 & 0 & 0 & 0 \\ 0 & 0 & 0 & 0 \\ 0 & 0 & (\mu-\varepsilon) & 0 \\ 0 & 0 & 0 & -r_{3} \delta_{1}\end{array}\right]$

In view of [19] and [22], the desired definitions for existence and uniqueness are defined as follows;

Definition 3.1. Let $C^{*}[0, T]$ be the class of continuous column vector $U(t)$ whose components $x, y, z, w \in C[0, T]$ are the class of continuous functions on the interval $[0, T]$. The norm of $U \in C^{*}[0, T]$ is given by

$$
\begin{gathered}
|| U \|=\sup _{t}\left|e^{-N t} x(t)\right|+\sup _{t}\left|e^{-N t} y(t)\right| \\
+\sup _{t}\left|e^{-N t} z(t)\right|+\sup _{t}\left|e^{-N t} w(t)\right| .
\end{gathered}
$$

When $t>\sigma \geq 0$, we write $C_{\sigma}^{*}[0, T]$ and $C_{\sigma}[0, T]$.

Definition 3.2. $U \in C^{*}[0, T]$ is a solution of the initial value problem (17), if

$(i)(t, U(t)) \in D, t \in[0, T]$ where $D=[0, T] \times K$,

$K=\left\{(x, y, z, w) \in R_{+}^{2}:|x| \leq l_{1},|y| \leq l_{2}|z| \leq l_{3},|w| \leq l_{4}\right\}$.

$$
\text { (ii) } U(t)
$$

satisfies (17).

Theorem 3.1.The initial value problem (17) has a unique solution $U \in C^{*}[0, T]$.

Proof. Let us write

$$
\begin{gathered}
I^{1-\alpha} \frac{d}{d t} U(t)=A U(t)+S(t) B U(t)+R(t) C U(t) \\
+N(t) D U(t)+Z(t) E U(t) .
\end{gathered}
$$

Operating with $I^{\alpha}$ we obtain

$$
U(t)=U(0)+I^{\alpha}(A U(t)+S(t) B U(t)
$$




$$
+R(t) C U(t)+N(t) D U(t)+Z(t) E U(t)) .
$$

Now let $F: C^{*}[0, T] \rightarrow C^{*}[0, T]$ be defined by

$$
\begin{gathered}
F U(t)=U(0)+I^{\alpha}(A U(t)+S(t) B U(t)+R(t) C U(t) \\
+N(t) D U(t)+Z(t) E U(t)) .
\end{gathered}
$$

Then

$$
\begin{aligned}
& e^{-N t}\|F U-F V\|= \\
& e^{-N t} I^{\alpha}(A(U(t)-V(t))+S(t) B(U(t)-V(t)) \\
& +R(t) C(U(t)-V(t))+N(t) D(U(t)-V(t))+ \\
& Z(t) E(U(t)-V(t)) \\
& \leq \frac{1}{\Gamma(\alpha)} \int_{0}^{t}(t-s)^{\alpha-1} e^{-N(t-s)}(U(s)-V(s)) e^{-N s}(A+a B+ \\
& b C) d s \\
& \leq \frac{(A+a B+b C)}{N^{\alpha}}\|U-V\| \int_{0}^{t} \frac{s^{\alpha-1}}{\Gamma(\alpha)} d s .
\end{aligned}
$$

This implies that

$$
\|F U-F V\| \leq \frac{\left(A+B l_{1}+C l_{2}+D l_{3}+E l_{4}\right)}{N^{\alpha}}\|U-V\| .
$$

If we choose $\mathrm{N}$ such that

$$
N^{\alpha}>A+B l_{1}+C l_{2}+D l_{3}+E l_{4}
$$

then we obtain

$$
\|F U-F V\|<\|U-V\|
$$

and the operator F given by (23) has a unique fixed point. Consequently, (22) has a unique solution $U \in C^{*}[0, T]$.From (22), we have

$U(t)$

$U(0)+\left(\frac{t^{\alpha}}{\Gamma(\alpha+1)}(A U(0)+S(0) B U(0)+R(0) C U(0))+\right.$ $N(0) D U(0)+Z(0) E U(0))+I^{\alpha+1}\left(A U^{\prime}(t)+S^{\prime}(t) B U(t)+\right.$ $S(t) B U^{\prime}(t)+R^{\prime} C U(t)+R(t) C U^{\prime}(t)+$

$\left.N^{\prime}(t) D U(t)+N(t) C U^{\prime}(t)+Z^{\prime} E U(t)+Z(t) E U^{\prime}(t)\right)$

$\frac{\text { and }}{d t}=\frac{t^{\alpha-1}}{\Gamma(\alpha)}(A U(0)+S(0) B U(0)+R(0) C U(0)+$ $N(0) D U(0)+Z(0) E U(0))+I^{\alpha}\left(A U^{\prime}(t)+S^{\prime}(t) B U(t)+\right.$ $S(t) B U^{\prime}(t)+R^{\prime} C U(t)+R(t) C U^{\prime}(t)+N(t) D U(t)+$ $\left.N(t) D U^{\prime}(t)+Z^{\prime} E U(t)+Z(t) E U^{\prime}(t)\right)$ $\Rightarrow e^{-N t}\left(\frac{U(t)}{d t}\right)=e^{-N t}\left[\frac{t^{\alpha-1}}{\Gamma(\alpha)}(A U(0)+S(0) B U(0)+\right.$ $R(0) C U(0)+N(0) D U(0)+Z(0) E U(0))$

$+I^{\alpha}\left(A U^{\prime}(t)+S^{\prime}(t) B U(t)+S(t) B U^{\prime}(t)+R^{\prime} C U(t)+\right.$ $R(t) C U^{\prime}(t)+N^{\prime}(t) D U(t)+N(t) D U^{\prime}(t)+Z^{\prime} E U(t)+$ $\left.\left.Z(t) E U^{\prime}(t)\right)\right]$

from which we can deduce that $U^{\prime} \in C_{\sigma}^{*}[0, T]$.Now, we have

$\frac{d U(t)}{d t}=\frac{d}{d t} I^{\alpha}(A U(t)+S(t) B U(t)+R(t) C U(t)+$ $N(t) D U(t)+Z(t) E U(t))$

$\Rightarrow \quad I^{1-\alpha} \frac{d U(t)}{d t}=I^{1-\alpha} \frac{d}{d t} I^{\alpha}(A U(t)+S(t) B U(t)+$ $R(t) C U(t)+N(t) D U(t)+Z(t) E U(t))$

$D^{\alpha} U(t)=A U(t)+S(t) B U(t)+R(t) C U(t)+$ $N(t) D U(t)+Z(t) E U(t)$

and
$U(0)=U_{0}+I^{\alpha}(A U(0)+S(0) B U(0)+R(0) C U(0)+$ $N(0) D U(0)+Z(0) E U(0))=U_{0}$. Therefore, this initial value problem is equivalent to the initial value probem (17).

\section{Analysis of the extinction of GBM by using Allee effect}

An important research for population models by Allee [28], who demonstrated that Allee effect occurs when population growth rate is reduced at low population size. It is well known that the logistic model assumes that per-capita growth rate declines monotonic when the density increase; however, it is shown that for population subjected to an Allee effect, per-capita growth rate gives a humped curve increasing at low density, up to a maximum intermediate density and then declines again [29]. Many theoretical and laboratory studies have demonstrated the importance of the Allee effect in dynamics of small populations, see for example [28 - 31].

Let us embed to (4) functions such as

$$
\left\{\begin{array}{l}
D^{\alpha}(S(t))=\left(p S(t)+r_{1} S(t)\left(K_{1}-\beta_{1} S(t)\right)-\gamma S(t) R(t)\right. \\
\left.-d_{1} S(t)-\tau_{1} S(t) N(t)\right)\left(\frac{S(t)}{E_{0}}-1\right) \\
D^{\alpha}(R(t))=\left(r_{2} R(t)\left(K_{2}-\beta_{2} R(t)\right)+\gamma S(t) R(t)\right. \\
\left.-d_{2} R(t)-\tau_{2} R(t) N(t)\right)\left(\frac{R(t)}{E_{1}}-1\right) \\
D^{\alpha}(N(t))=\mu N(t) Z(t)-\varepsilon Z(t) N(t)-d_{3} N(t) \\
D^{\alpha}(Z(t))=r_{3} Z(t)\left(K_{3}-\delta_{1} Z(t)\right)-\mu N(t) Z(t) \\
+\varepsilon Z(t) N(t)-d_{4} Z(t)
\end{array}\right.
$$

where $>\quad 0$

and $(S(0), R(0), N(0), Z(0))=\left(S_{0}, R_{0}, N_{0}, Z_{0}\right) . \quad E_{0}$ and $E_{1}$ represents the Allee threshold of the populations $S(t)$ and $R(t)$, respectively. The equilibrium point that will be considered in this section is $\Lambda_{3}=\left(E_{0}, E_{1}, B, \frac{d_{3}}{\mu-\varepsilon}\right)$. The characteristic equation of (24) is

$$
\begin{gathered}
P(\lambda)=\left(\lambda-\frac{\left(p E_{0}+r_{1} E_{0}\left(K_{1}-\beta_{1} E_{0}\right)-\gamma E_{0} E_{1}-d_{1} E_{0}-\tau_{1} E_{0} B\right)}{E_{1}}\right) \\
\times\left(\lambda-\frac{\left(r_{2} E_{1}\left(K_{2}-\beta_{2} E_{1}\right)+\gamma E_{0} E_{1}-d_{2} E_{1}-\tau_{2} E_{1} B\right)}{E_{1}}\right) \\
\left(\lambda^{2}-\left(\frac{\left(r_{3} K_{3}-d_{4}-(\mu-\varepsilon) B\right)-2 r_{3} \delta_{1} d_{3}}{\mu-\varepsilon}\right) \lambda(\mu-\varepsilon) B\right)=0,
\end{gathered}
$$

The following theorems will be given without the proofs, which can be proven in view of Section 2 and Section 3.

Theorem 4.1. Let $\left(E_{0}, E_{1}, B, \frac{d_{3}}{\mu-\varepsilon}\right)$ be the positive equilibrium point of system (24). The following statements are true.

(a) Assume that $K_{1}>\frac{r_{1} \beta_{1}\left(d_{2}+\tau_{2} B\right)-p \gamma}{r_{1} \gamma}$ and $K_{3}<\frac{d_{4}+(\mu-\varepsilon) B}{r_{1}}$ hold, such that $p<\frac{r_{1} \beta_{1}\left(d_{2}+\tau_{2} B\right)}{\gamma}$. If 


$$
E_{1}>\frac{K_{2}}{\beta_{2}} \text { and } \frac{p+r_{1} K_{1}}{r_{1} \beta_{1}}<E_{0}<\frac{d_{2}+\tau_{2} B}{\gamma},
$$

then all roots of (25) have are real or complex conjugates with negative real parts, which means that $\Lambda_{3}$ is locally asymptotically stable.

(b) Assume that $K_{1}>\frac{r_{1} \beta_{1}\left(d_{2}+\tau_{2} B\right)-p \gamma}{r_{1} \gamma}$ and $\frac{8 \delta_{1} d_{3}}{\mu-\varepsilon}>K_{3}>\frac{\left(d_{2}+\tau_{2} B\right) \beta_{2}}{\gamma}>\frac{4 \delta_{1} d_{3}}{\mu-\varepsilon}$ hold, such that $p<\frac{r_{1} \beta_{1}\left(d_{2}+\tau_{2} B\right)}{\gamma}$. Furthermore, suppose that

$$
\begin{gathered}
\frac{(\mu-\varepsilon) d_{4}+(\mu-\varepsilon)^{2} B}{8 \delta_{1} d_{3}}<r_{3}<\frac{(\mu-\varepsilon) d_{4}+(\mu-\varepsilon)^{2} B}{4 \delta_{1} d_{3}}, \\
\frac{\mu-\varepsilon}{r_{3} \delta_{1}}>\sqrt{\frac{d_{3}}{(\mu-\varepsilon) B}}
\end{gathered}
$$

and

$\left|\tan ^{-1}-\sqrt{4} d_{3}(\mu-\varepsilon)-\left(\frac{\left(r_{3} K_{3}-d_{4}-(\mu-\varepsilon) B\right)(\mu-\varepsilon)-2 r_{3} \delta_{1} d_{3}}{\mu-\varepsilon}\right)^{2}\right|$ $/\left(\frac{\left(r_{3} K_{3}-d_{4}-(\mu-\varepsilon) B\right)(\mu-\varepsilon)-2 r_{3} \delta_{1} d_{3}}{)}\right)>\frac{\alpha \pi}{2}$ hold. If

$$
E_{1}>\frac{K_{2}}{\beta_{2}} \text { and } \frac{p+r_{1} K_{1}}{r_{1} \beta_{1}}<E_{0}<\frac{d_{2}+\tau_{2} B}{\gamma}
$$

then (25) has has two real or complex conjugates with negative real parts and two complex conjugate with positive real parts, where $\Lambda_{3}$ is locally asymptotically stable.

Theorem 4.2.Let $p>d_{1}, r_{2}>\frac{d_{2}}{K_{2}}, r_{3}>\frac{d_{3}}{K_{3}}$ and $\mu>\varepsilon$. System (24) has a unique solution in

$W=\left\{(S, R, N, Z) \in R_{+}^{4}:|S| \leq E_{0},|R| \leq E_{1},|N| \leq l_{3},|Z| \leq l_{4}\right\}$,

if

$$
\begin{gathered}
\frac{3 r_{1} \beta_{1} E_{0}+p+r_{1} K_{1}+3 \gamma E_{1}-d_{1}+3 \tau_{1} l_{3}}{N^{\alpha}}<1, \\
\frac{3 r_{2} \beta_{2} E_{1}+r_{2} K_{2}+\gamma E_{0}-d_{2}+3 \tau_{2} l_{3}}{M^{\alpha}}<1, \\
\frac{(\mu-\varepsilon) l_{4}-d_{3}}{P^{\alpha}}<1
\end{gathered}
$$

and

$$
\frac{2 \delta_{1} r_{3} l_{4}+r_{3} K_{3}+(\mu+\varepsilon) l_{3}-d_{4}}{Q^{\alpha}}<1 .
$$

Conclusion If $S(t)<E_{0}$ and $R(t)<E_{0}$ for all $t>0$, where $(S(t), R(t), N(t), Z(t))$ ia a positive solution of system (24), then $S(t)$ and $R(t)$ go to zero while $t \rightarrow \infty$.

ExampleThe values of parameter of (4) are mainly so selected as given in [8] and in view the conditions of the

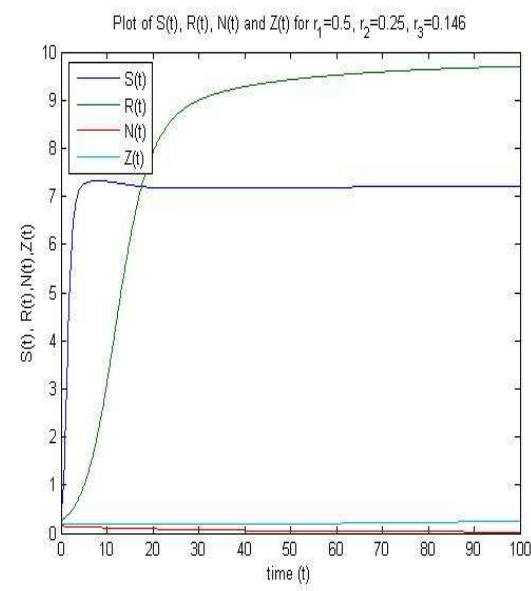

Fig. 1: The asymptotic behavior of the solutions of system (4)

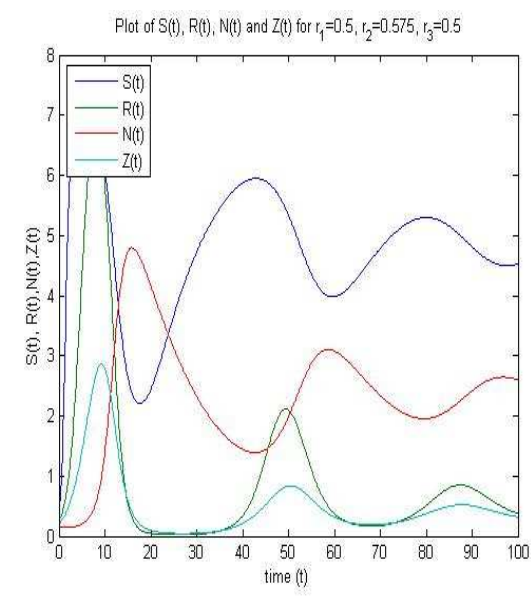

Fig. 2: Interaction of the tumor-IS populations

theorems. Information of activated macrophases and macrophases are obtained from [13]. Therefore, the table is given as follows;

Division rate of the sensitive cells: $p=0.192$

Carriying capacity of the negrotic and sensitive cells together: $K_{1}=4.704$

Carriying capacity of the resistant tumor population: $K_{2}=1.232$

Mutation rate of the sensitive cells to resistant cells: $\gamma \in\left[10^{-5}, 10^{-2}\right]$

Logistic population rate of sensitive cell population: $\beta_{1} \in[0.5,0.95]$

Logistic population rate of resistant cell population: $\beta_{2} \in[0.05,0.2]$

Causes of drug treatment to the sensitive cells: $d_{1}=0.6$

Causes of drug treatment to the resistant cells: $d_{2}=0.006$ Destroying rate caused from the activated macrophages to 


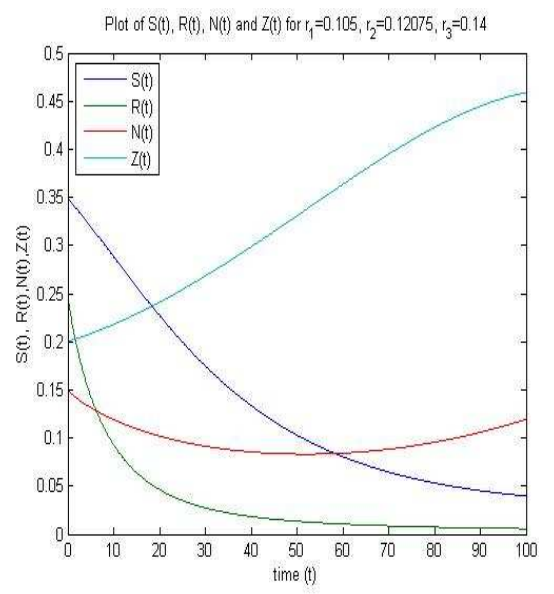

Fig. 3: The asymptotic behavior of the solutions of system (24)

the sensitive cells: $\tau_{1}=0.3$

Destroying rate caused from the activated macrophages to the resistant cells: $\tau_{2}=0.03$

Activation rate of the macrophages: $\mu=0.201$

Deactivation rate of the macrophages: $\varepsilon=0.01$

Logistic population rate of the macrophages: $\delta_{1} \in[0.05,0.2]$

Carrying capacity of the macrophages: $K_{3} \in[0.11,1.17]$

Natural death of macrophages: $d_{3}=0.07$

Natural death of active macrophages: $d_{4}=0.07$.

The population rates of both tumor cells are different, where here in Figure 1 and Figure 2 is taken $(1.05) * r=r_{2}$. The growth rate of the macrophage is $r_{3}$. For this example, the mutation rate is selected as $\gamma=0.01$. The initial conditions are $S(0)=0.35, R(0)=0.25, N(0)=0.15$, and $Z(0)=0.25$. Figure 1 shows the behavior of the system that hold the conditions of Theorem 2.1/(a).The growth rates are $r_{1}=0.5, r_{2}=0.525$ and $r_{3}=0.0146$. It is shown in this graph that a local asymptotic behavior about the equilibrium point $\mu_{2}$ occur, where $\alpha=0$.9.In Figure 2, we have an interaction behavior of the solutions, where $r_{1}=0.5, r_{2}=0.525, r_{3}=0.5, K_{3}=1.17$ and $\alpha=0.98$. A local asymptotic behavior of the solutions for $\alpha<0.88$ is obtained in Figure 3, where $r_{1}=0.105, r_{2}=0.1207, r_{3}=0.14$. The relation between the growth rates of the tumors are here (1.15) $* r=r_{2}$.

\section{Acknowledgement}

This work is supported by the Scientific Research Center at the Erciyes University with the project code FBA-124137.

\section{References}

[1] A. El-Gohary, Chaos and optimal control of cancer selfremission and tumor system steady states, Chaos, Solutions and Fractals, 37, 1305-1316 (2008).

[2] E. C. Holland, Glioblastoma multiforme: The Terminator, Proc. of the Nat. Acad. of Sci. , 97, 6242-6244 (2000). E. C. Holland, Glioblastoma multiforme: The Terminator, Proc. of the Nat. Acad. of Sci., 97, 6242-6244 (2000).

[3] Y. A. Yung, J. R. Shapiro and W.R. Shapiro, Heterogeneous chemosensitivities of subpopulations of human glioma cells in culture, Cancer Res., 42, 992-998 (1982).

[4] W. Paulus and J. Peiffer, Intratumoral histologic heterogeneity of gliomas, A quantitative study, Cancer, 64, 442-447 (1989).

[5] R. A. Berkman et al., Clonal composition of glioblastoma multiforme, Journal of Neurosurgery, 77, 432-437 (1992).

[6] A. J. Coldman and J. H. Goldie, A mathematical model for relating the drug sensitivity of tumors to their spontaneous mutation rate, Cancer Treat. Rep., 63, 1727-1731 (1979).

[7] J. C. Panetta, A mathematical model of drug resistance: Heterogeneous tumors, Math. Biosci., 147, 41-61(1998).

[8] B. G. Birkhead et al., A mathematical model of the development of drug resistance to cancer chemotherapy, Europ. J. of Cancer and Clinical Oncology, 23, 1421-1427 (1987).

[9] J. E. Schmitz, A. R. Kansal and S. Torquato, A cellular Automaton of Brain Tumor Treatment and Resistance, J. of Theoret. Med., 4, 223-239 (2002).

[10] H. P. de Vladar and J. A. Gonzalez, Dynamic response of cancer under the influence of immunological activity and therapy, J. of Theoret. Biol., 227, 335-348 (2004).

[11] A. El-Gohary, The chaos and optimal control of cancer model with complete unkown parameters, Chaos, Solutions and Fractals, 42, 2865-2874 (2009).

[12] A. Merola et al., An insight into tumor dormancy equilibrium via the analysis of its domain of attraction, Biomedical Signal Processing and Control, 3, 212-219 (2008).

[13] B. Mukhopadhyay, R. Bhattacharyya, Temporal and spatiotemporal variations in a mathematical model of macrophage-tumor interaction, Nonlinear Analysis: Hybrid Systems, 2, 819-831 (2008).

[14] A. S. Perelson, D. E. Kirschner, R. De Boer, Dynamics of HIV infection of $(C D 4)^{+}$T-cells, Mathematical Bioscience, 114, 81-125 (1993).

[15] R. V. Culshaw, S. Ruan, A delay-differential equation model of HIV infection of $(C D 4)^{+}$T-cells, Mathematical Bioscience, 165, 27-39 (2000).

[16] A. S. Perelson, P. W. Nelson, Mathematical Analysisof HIV1 dynamics in vivo, SIAM Review, 41, 3-44 (1993).

[17] D. E. Kirschner, Using Mathematics to understand HIV immune dynamics, Notice of the American Mathematical Society, 43, 191-202 (1996).

[18] E. Ahmed, A. S. Elgazzar, On fractional order differential equations model for nonlocal epidemics, Physica A, 379, 607-614 (2007).

[19] E. Ahmed, A. M. A. El-Sayed, H. A. A. El-Saka, Equilibrium points, stability and numerical solutions of fractional order predator-prey and rabies models, Journal of Mathematical Analysis and Applications, 325, 542-553 (2007). 
[20] M. P. Lazarevic, Finite time stability analysis of $(P D)^{\alpha}$ fractional ccontrol of robotic time delay systems, Mechanics Research Communications, 33, 269-279 (2006).

[21] T. J. Anastasio, The fractional order Dynamics of bainstem vestibulo-oculomotor neurons, Biological Cybernetics, 72, 69-79 (1994).

[22] A. M. A. El-Sayed, A. E. M. El- Mesiry, H. A. A. ElSaka, On the fractional order logistic equation, Applied Mathematics Letters, 20, 817-827 (2007).

[23] K. S. Cole, Electric conductance of biological systems, in: Proc. Cold Sring Harbor Symp. Quant. Biol., Cold Sring Harbor, New York, 107-116 (1993).

[24] V. D. Djordjevic, J. Jaric, B. Fabry, J. J. Fredberg, D. Stamenovic, Fractional derivatives embody essential features of cell rheological behavior, Annals of Biomedical Engineering, 31, 692-699 (2003).

[25] I. Podlubny, Fractional Differential Equations, Academic Press, New York, (1999).

[26] D. Matignon, Stability results for fractional differential equations with applications to control processing, Computational Eng. in Sys. Appl., Lille, France, 2, 963, (1996).

[27] E. Ahmed, A. M. A. El-Sayed, H. A. A. El-Saka, On some Roth-Hurwitz conditions for fractional order differential equations and their applications in Lorenz, Rssler, Chu and Chen systems, Physics Letters A, 358, 1-4 (2006).

[28] W. C. Allee, Animal Aggregations: A Study in General Sociology. University of Chicago Press, Chicago, IL, (1931).

[29] W. C. Allee, Animal Aggregations: A Study in General Sociology. University of Chicago Press, Chicago, IL, (1931).

[30] G. Wang, X-G., Liang, F.-Z., Wang, The competitive dynamics of populations subject to an Allee Effect, Ecological Modelling, 124, 183-192 (1999).

[31] M. A.Asmussen, Density-dependent selection II. The Allee effect. Am. Nat., 114, 796-809 (1979).

[32] M. Al-Refai, On the fractional derivatives at extreme points, Electronic Journal of Qualitative Theory of Differential Equations, 55, 1-5 (2012).

[33] M. Al-Refai, Basic results of nonlinear eigenvalue problems of fractional order, Electronic Journal of Differential Equations, 191, 1-12 (2012).

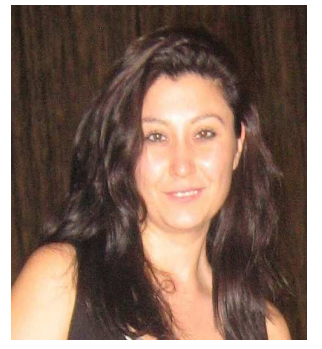

Fatma Bozkurt Assis. Prof. Dr. Fatma Bozkurt was born in Germany in 1979. Since 1994 she lives in Turkey. She studied Mathematics at the Erciyes University (1998-2002), and has two MSci, which one is about Applied Mathematics ( The periodic character of the solutions of some difference equation, Erciyes University, Turkey, 2002-2005) and the other about Mathematics Education (Hacettepe University, Turkey, 2003-2005). Her $\mathrm{PhD}$ thesis is about the population dynamics of single species (Stability Analysis for Differential and Difference Equations in Population Models, Erciyes University, Turkey, 2006-2010). She worked from 2005 until 2010 as Research Assistant at the Erciyes University and got the Assis. Prof. Dr. title in 2010. Since 2010 she is working as Assis. Prof. Dr. at the Erciyes University in Kayseri (Turkey). Her research fields are about the dynamics of species and especially about modeling and analyzing the brain tumor GBM. She is head of some projects about modeling GBM tumor and the epidemic disease HIV/AIDS (FBA-12-4137, FBY-12-3993) and is Researcher at the project FBA-11-3597 